Revue d'archéologie préhistorique

\title{
La Chaise-de-Vouthon (Charente), fouilles P. David (1936-1961) et fouilles A. Debénath (1967-1983) : un exemple de débitage bipolaire sur enclume
}

La Chaise-de-Vouthon (Charente), excavations P. David (1936-1961) and A.

Debénath (1967-1983): an example of bipolar-on-anvil debitage

\section{Karine Matilla}

\section{OpenEdition \\ Journals}

Édition électronique

URL : http://journals.openedition.org/paleo/1907

DOI : $10.4000 /$ paleo.1907

ISSN : 2101-0420

Éditeur

SAMRA

Édition imprimée

Pagination : 63-74

ISSN : $1145-3370$

\section{Référence électronique}

Karine Matilla, « La Chaise-de-Vouthon (Charente), fouilles P. David (1936-1961) et fouilles A. Debénath (1967-1983) : un exemple de débitage bipolaire sur enclume », PALEO [En ligne], Numéro spécial |

2009-2010, mis en ligne le 23 avril 2012, consulté le 07 juillet 2020. URL : http://

journals.openedition.org/paleo/1907 ; DOI : https://doi.org/10.4000/paleo.1907

\section{(c) (i) $\ominus$}

PALEO est mis à disposition selon les termes de la licence Creative Commons Attribution - Pas d'Utilisation Commerciale - Pas de Modification 4.0 International. 


\title{
LA CHAISE-DE-VOUTHON (Charente), FOUILLES P. DAVID (1936-1961) ET FOUILLES A. DEBÉNATH (1967-1983) : UN EXEMPLE DE DÉBITAGE BIPOLAIRE SUR ENCLUME
}

\author{
Karine MATILLA ${ }^{(1)}$
}

\begin{abstract}
Résumé : La grotte de La Chaise, en Charente, a été fréquentée durant près de 100000 ans. Le matériel étudié, sur galet de quartz, représente un total de 13148 objets. L'analyse technologique met en avant l'application de méthodes de débitage organisées, Levallois et discoïdes, ainsi que la pratique d'un débitage plus opportuniste, bipolaire sur enclume notamment. II s'agit, d'une part, d'une technique employée pour entamer les galets destinés ensuite à être débités par percussion directe classique, mais surtout d'une méthode de débitage permettant d'obtenir des éclats en série pour un besoin immédiat. La présence de plusieurs méthodes de débitage relevant de conceptions techniques et technologiques différentes et indépendantes révèle la richesse des connaissances des tailleurs, et leur capacité d'adaptation en fonction de leurs besoins. De plus, il s'agit d'un comportement techno-économique particulier.
\end{abstract}

Mots-clés : OIS 6 à 5.3, Paléolithique moyen, La Chaise-de-Vouthon, Charente, France, industrie lithique, techno-typologie et techno-économie, comportement humain.

\begin{abstract}
La Chaise-de-Vouthon (Charente), excavations P. David (1936-1961) and A. Debénath (1967-1983) : an example of bipolar-on-anvil debitage. The cave of La Chaise, in Charente, has been occupied while about 100000 years. The material studied, on quartz pebble, represents 13148 objects. The technologic analysis shows the practice of organised methods of debitage, Levallois and discoïdal, and the practice of a more opportunist debitage, bipolar on anvil. It is a technique used to split pebbles debited next by classic percussion, but first of all, it's a method of debitage who permits to obtain series of flakes for an immediate need. The presence of several methods of debitage from different and independent technic and technological conceptions reveals the important knowledge of the prehistorics, and their capacity of adaptation according their needs. Moreover, it is a particular techno-economic behaviour.
\end{abstract}

Key-words: OIS 6 to 5.3, Middle Palaeolithic, La Chaise-de-Vouthon, Charente, France, lithic industry, techno-typology and technoeconomy, human behaviour.

\section{INTRODUCTION}

La grotte de La Chaise-de-Vouthon, située à quelques kilomètres d'Angoulême, en Charente (fig. 1), est un complexe d'habitats constitué des abris Suard et BourgeoisDelaunay, ainsi que de la grotte Duport. Ces cavités communiquent entre elles par des couloirs (fig. 2). II a été fréquenté à l'abri Suard puis à l'abri Bourgeois-Delaunay, sans interruption, de la fin du stade isotopique 6 à l'interstade 5.3, les deux remplissages étant séparés par l'interglaciaire du stade 5.5. En dehors des silex, le matériel est constitué de galets alluviaux d'origine locale. Par commodité, mais en ayant bien conscience de l'existence de silex sous cette forme, nous dénommerons ici cette composante « industrie sur galet ». La nature pétrographique de ce matériel est à $93 \%$ représentée par des quartzites dans lesquels sont inclus quelques rares quartz. Le reste concerne les roches magmatiques : granite, microgranite, et basalte (tab. 1 et fig. 3). Le matériel étudié ici est issu des fouilles de P. David et d'A. Debénath et représente un total de 13148 objets, 

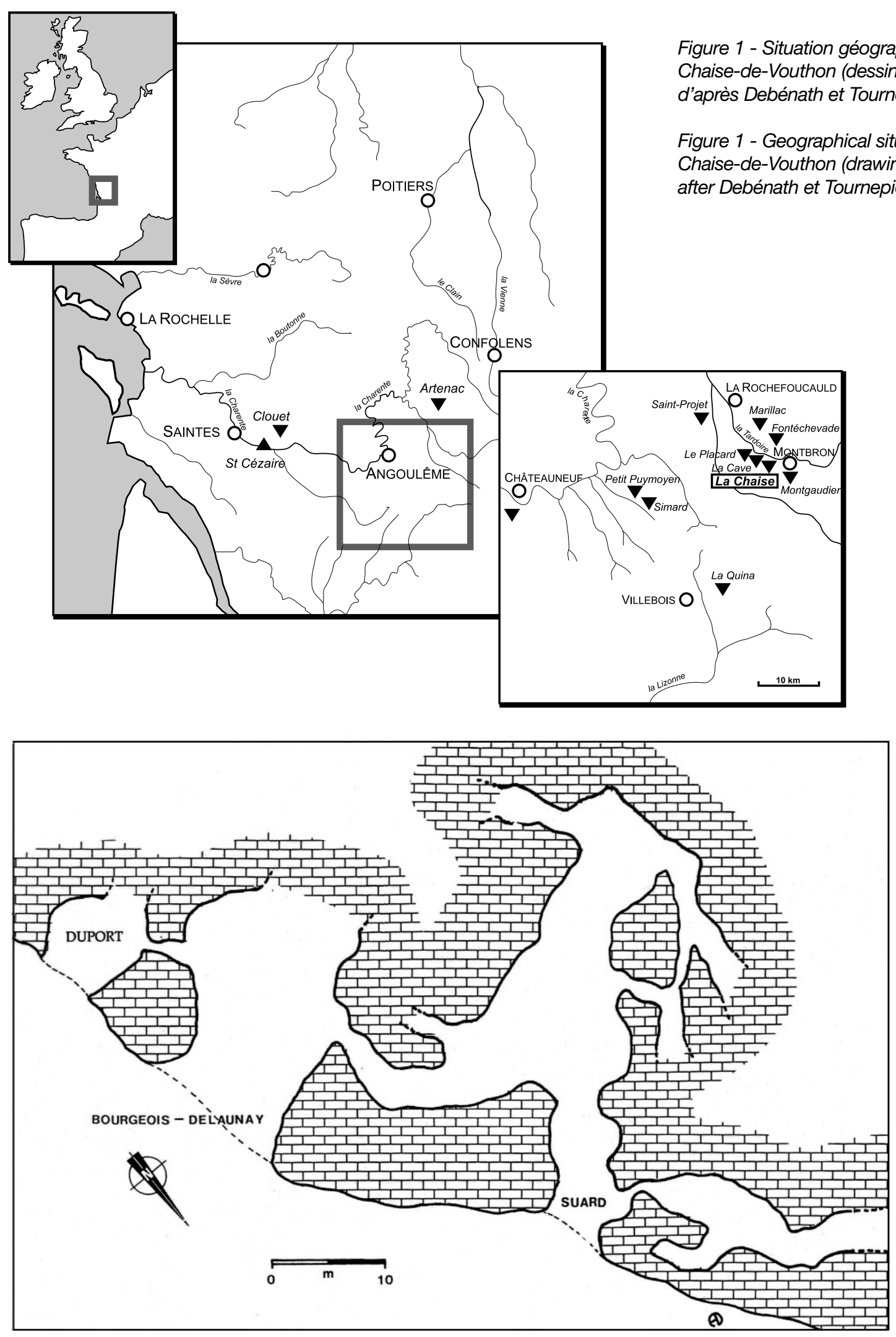

Figure 2 - Plan de La Chaise-de-Vouthon (in Debénath 1974).

Figure 2 - Plan of La Chaise-de-Vouthon. 


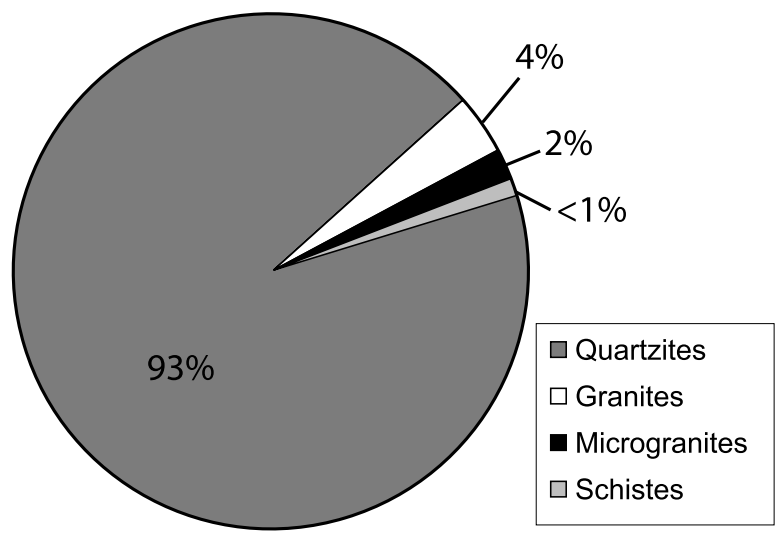

\begin{tabular}{|l|r|}
\hline \multicolumn{1}{|c|}{ Roche } & Nombre \\
\hline Quartzite & 12247 \\
\hline Granites & 566 \\
\hline Microgranites & 276 \\
\hline Schistes & 59 \\
\hline Total & 13148 \\
\hline
\end{tabular}

Tableau 1 - Proportion pétrographique du matériel étudié.

Table 1 - Petrologic proportion of the studied industry.

Figure 3 - Proportion pétrographique du matériel étudié.

Figure 3 - Petrologic proportion of the studied industry.

\begin{tabular}{|c|c|c|}
\hline & $\begin{array}{c}\text { Matériel en silex } \\
\text { (étude A. Delagnes) }\end{array}$ & $\begin{array}{c}\text { Matériel sur galet } \\
\text { (matériel K. Matilla) }\end{array}$ \\
\hline \multicolumn{3}{|l|}{ Suard } \\
\hline couche 54 & - & 54 \\
\hline couche 53 & 178 & 118 \\
\hline couche 52 & 479 & 312 \\
\hline couche 51 & 896 & 618 \\
\hline couche 50 & 69 & 58 \\
\hline couche $49 / 48$ & 24 & 30 \\
\hline Remanié & - & 241 \\
\hline Total & $\begin{array}{c}1646 \\
\text { (soit 53,5\%) }\end{array}$ & $\begin{array}{c}1431 \\
\text { (soit } 46,5 \% \text { ) }\end{array}$ \\
\hline \multicolumn{3}{|l|}{ Bourgeois-Delaunay } \\
\hline couche 12 & - & 5 \\
\hline couche 10 & 574 & 630 \\
\hline couche 9 & 2829 & 1300 \\
\hline couche $8^{6}$ & 1497 & 329 \\
\hline couche 8 & 1031 & 863 \\
\hline Total & $\begin{array}{c}5931 \\
\text { (soit } 68 \% \text { ) }\end{array}$ & $\begin{array}{c}2798 \\
\text { (soit } 32 \% \text { ) }\end{array}$ \\
\hline Total des deux abris & $\begin{array}{c}7577 \\
\text { (soit 64,2\%) }\end{array}$ & $\begin{array}{c}4229 \\
\text { (soit } 35,8 \% \text { ) }\end{array}$ \\
\hline
\end{tabular}

Tableau 2 - Décompte du matériel en silex et du matériel sur galet, fouilles A. Debénath.

Table 2 - Silex and pebble industry count, A. Debénath's excavations. 
8919 provenant des fouilles de David, 4229 des fouilles d'A. Debénath. Seul le matériel en silex provenant des fouilles d'A. Debénath ayant été étudié (Debénath 1974 ; Delagnes 1992), nous ne pouvons comparer la proportion des deux matériaux qu'avec cette partie du matériel. Ainsi, ce matériel sur galet de toute nature pétrographique, hors silex, représente $46,5 \%$ de l'assemblage total de l'abri Suard et 35,8 \% de celui de Bourgeois-Delaunay (tab. 2).

Cette composante représente donc une part importante de la panoplie lithique totale et son étude est indispensable à la compréhension du comportement techno-typologique et techno-économique des hommes. Ainsi, il semble complémentaire de l'assemblage en silex. Des changements comportementaux ont été observés entre les panoplies des deux abris, Suard et Bourgeois-Delaunay (Matilla 2004).

Malgré cela, la percussion bipolaire sur enclume, qui nous concerne ici, est omniprésente tout au long de l'occupation des sites et ne semble pas participer à cette évolution, constituant une forme de continuité technologique.

Quels ont été les objectifs des tailleurs ? Est-il possible d'évaluer les méthodes et techniques mises en œuvre? Quel est le statut du débitage sur enclume dans les schémas opératoires des industries de La Chaise-de-Vouthon?

\section{La percussion bipolaire sur enclume : technique d'en- tame, méthode de débitage...}

L'analyse technologique met en avant l'application de méthodes de débitage organisées, Levallois et discoïdes, ainsi que l'emploi, plus opportuniste, de la percussion bipo- laire sur enclume. Celle-ci, dite aussi « percussion bipolaire simultanée » (Jaubert et Mourre 1996), présente de manière régulière tout au long des occupations des deux abris, peut donc être employée pour atteindre deux objectifs : l'initialisation du débitage ou une production à part entière.

\section{L'initialisation}

La percussion bipolaire sur enclume peut être une technique d'entame de galets qui n'offrent aucun angle adéquat au débitage par percussion directe simple (Mourre 1996). II s'agit de galets arrondis (Bordes 1961) ou ovalaires posés sur l'enclume et percutés au percuteur dur. Deux points d'impacts opposés sont alors visibles sur le " galet-nucléus » et sur l' « éclat » obtenu. L'écrasement du point d'impact imprimé par le percuteur est beaucoup plus marqué que le point d'impact de contrecoup provoqué par l'enclume sur l'extrémité opposée (Mourre 1997). Cette dernière caractéristique est toutefois essentielle pour reconnaître l'emploi de cette méthode sur les galets ainsi fracturés (Duran 2002 ; Matilla 2002). Les entames débitées peuvent, selon leur dimension, servir comme supports de nucléus Levallois ou discoïdes, comme supports d'outil ou être abandonnées.

Ainsi, la percussion bipolaire sur enclume peut être une simple technique d'entame des galets destinés ensuite à être débités par percussion directe classique, mais aussi une méthode de débitage à part entière, bien qu'opportuniste, permettant d'obtenir des éclats en série pour un besoin immédiat.

\section{La production}

Le débitage s'effectue le plus souvent à partir d'une seule face. Les éclats sont débités parallèlement entre eux, en
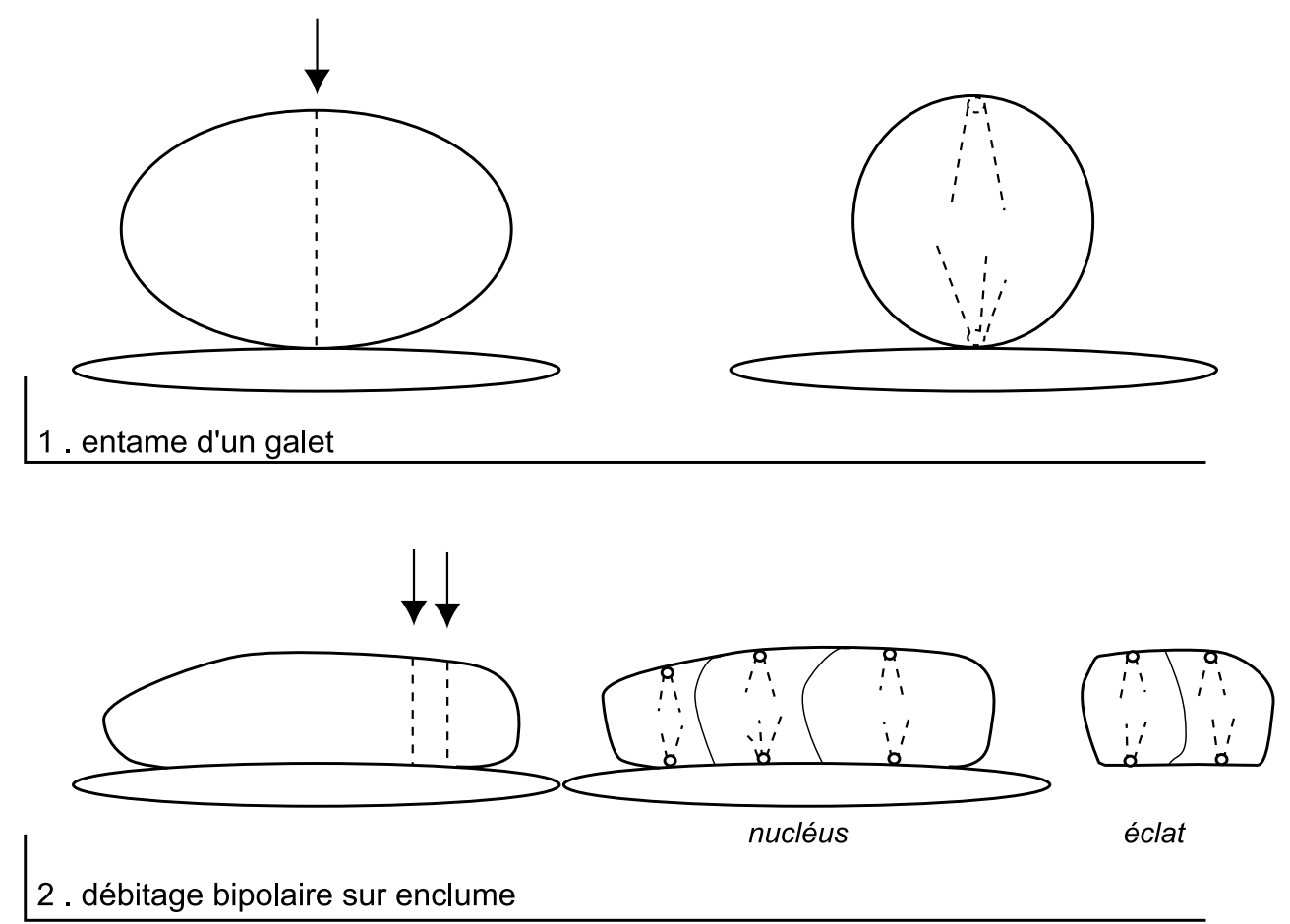

Figure 4 - Percussion bipolaire sur enclume (1) et débitage bipolaire sur enclume (2).

Figure 4 - Bipolar percussion (1) and debitage on anvil (2). 
suivant les nervures guides créées au fur et à mesure. Le nombre d'enlèvements n'est jamais très important dans le cas présent, de l'ordre de trois ou quatre. Lorsque des accidents interviennent tels que des réfléchissements ou des fractures, un autre plan de frappe, orthogonal ou opposé, peut être choisi. Le comportement est dans ce cas assez opportuniste, en fonction des plans de frappe, des angles, des surfaces de débitage disponibles (fig. 4).

Malgré les caractéristiques technologiques particulières des éclats obtenus, il n'est pas toujours aisé de les identifier. En effet, ils sont souvent cassés, fracturés par des accidents de type Siret (Matilla 2001), ce qui limite les possibilités d'identification de leurs caractéristiques, notamment le double point d'impact. De plus, ce sont des éclats souvent néocorticaux tels que ceux obtenus lors du décorticage des galets de manière directe. Afin d'attester la présence de cette technique de débitage et celle d'éclats entiers présentant clairement deux points de percussion synchrones et dans un même axe, la présence de nucléus bipolaires est donc nécessaire (Kuijt et al. 1995).

Selon F. Bordes, « II est difficile par ce procédé de savoir d'avance quel éclat on obtiendra et il n'est guère intéressant que dans le cas de matières très dures, telles que le quartz. » (Bordes 1947 : 16). Or, les études successives attestent désormais que la percussion sur enclume est une méthode de débitage à part entière, " qui permet d'obtenir d'importantes séries récurrentes d'éclats prédéterminés. " "Les produits recherchés présentent des caractéristiques morphotechniques déterminées par les enlèvements antérieurs : ils sont donc prédéterminés au sens premier de ce terme. » (Mourre 2004). Toutefois, cette prédétermination dépend, comme pour les autres méthodes de débitage d'ailleurs, et davantage même dans le cas de celle-ci, dont la percussion est relativement violente, de la qualité du matériau employé et de sa réaction au choc de percussion. Les accidents de débitage, fractures de type Siret notamment, sont beaucoup plus fréquents avec cette méthode comme le montrent les expérimentations. La prédétermination du résultat est par conséquent limitée à partir du matériau utilisé à La Chaise.

\section{Expérimentations}

Dans le cadre de l'étude d'industries lithiques en quartz/quartzite, les expérimentations sont nécessaires pour une bonne appréhension du matériau et une étude correcte du matériel. Elles permettent d'observer les réactions de la matière selon le mode percussion, classique ou sur enclume, de comprendre les techniques et méthodes de débitage choisies par les artisans préhistoriques, d'évaluer leur dextérité et la pertinence de leur choix.

Les galets utilisés ici proviennent d'une carrière proche de La Rochefoucauld, à huit kilomètres environ au nord de La Chaise. Les coupes ont mis au jour la terrasse du Lérat, une des anciennes terrasses alluviales de la Tardoire. Les galets utilisés, plusieurs centaines, sont de natures pétrographiques (quartz, quartzite, granite, microgranite, basalte), de morphologies (quadrangulaire, triangulaire, ovalaire), de poids (80 à $400 \mathrm{~g}$ en moyenne) et de dimensions (60 à 120 $\mathrm{mm}$ de long en moyenne) similaires aux objets archéologiques.

Le débitage bipolaire sur enclume est effectué soit pour entamer des galets, soit pour le débitage de galets volumineux et de morphologie inadaptée au débitage classique. Les percuteurs utilisés sont lourds, $700 \mathrm{~g}$ environ, afin que le poids accentue la force fournie. Quelques éclats longitudinaux unipolaires peuvent être débités mais les accidents de type Siret notamment, sont fréquents. Sans revenir ici sur le détail de ces expérimentations et sur leurs résultats (Matilla 2004), ce que nous retiendrons, c'est que l'obtention d'éclats en série, utilisables (fig. 5) nécessite de la part de l'artisan une véritable maîtrise technique, permettant d'exclure les qualificatifs «peu investi» ou «expédients», généralement associé à cette forme de débitage.

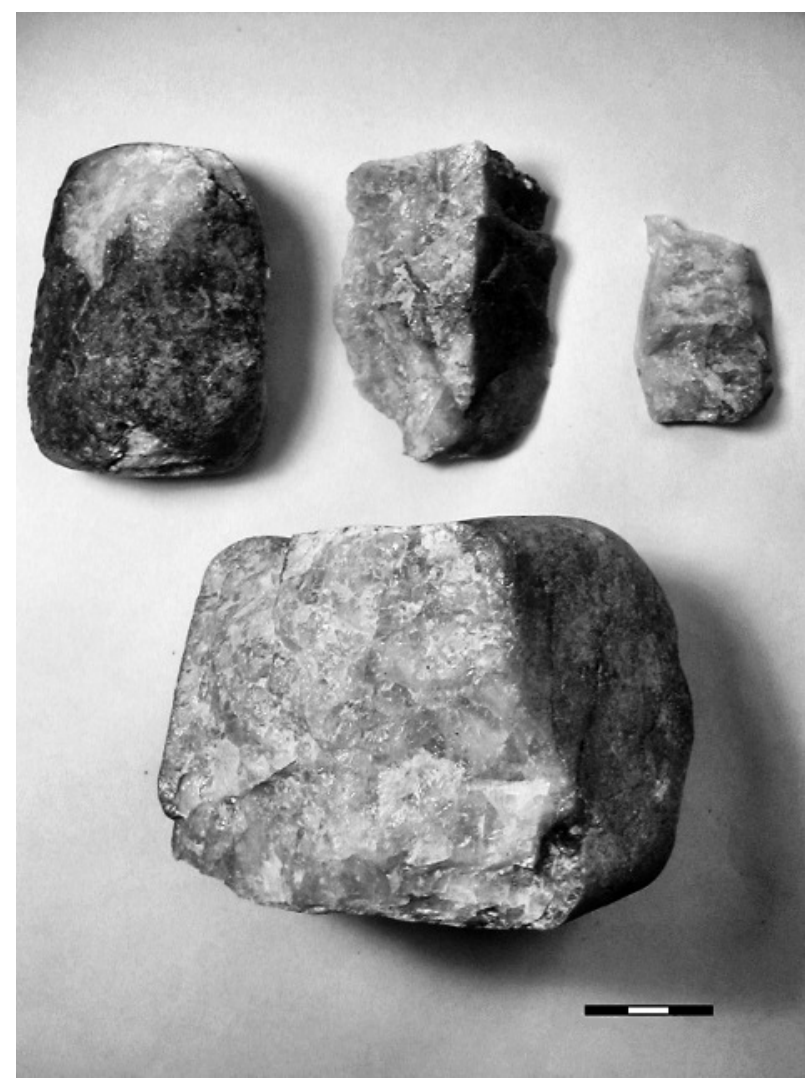

Figure 5 - Produits d'expérimentation, débitage bipolaire sur enclume : en haut, éclats bipolaires sur enclume; en bas, nucléus bipolaire sur enclume (quartzite).

Figure 5 - Products obtained by experimentation, debitage on anvil : above, bipolar flakes on anvil ; bipolar core on anvil (quartzite). 


\section{Remontages}

Les remontages qui peuvent être découverts au sein du matériel archéologique sont aussi très importants. Ils viennent compléter les expériences et confirmer les hypothèses émises. II s'agit ici de remontages découverts pour la plupart dans le matériel issu des fouilles d'A. Debénath à l'abri Suard, dont le remplissage stratigraphique a moins souffert que celui de l'abri Bourgeois-Delaunay qui a subi un affaissement des couches sans doute lié à des phénomènes de vidange. Les exemples présentés sont essentiellement des galets percutés sur enclume avec l'éclat correspondant. La vue supérieure des galets permet d'observer les points d'impact très marqués dus à l'emploi du percuteur dur. En vue de face, le point d'impact de contrecoup, aussi bien prononcé, est visible (fig. $6 \mathrm{n}^{\circ} 1 \mathrm{a}$. et 1b.). L'éclat obtenu peut être fracturé transversalement, dans sa partie médiane, au niveau de la rencontre de l'onde de choc de la percussion et de celle réfléchie par l'enclume, ou longitudinalement, et il s'agit alors d'un ou plusieurs fragments de type Siret (fig. $6 \mathrm{n}^{\circ} 2$ ).

\section{La percussion bipolaire sur enclume à La Chaise-de- Vouthon}

Que ce soit à l'abri Suard ou à l'abri Bourgeois-Delaunay, la percussion sur enclume est employée régulièrement comme méthode de débitage, parallèlement aux autres méthodes, Levallois et discoïdes. Elle représente environ $25 \%$ de la production du matériel sur galet. Les nucléus, mis en place sur des galets ne présentant pas d'angles de frappe adéquats pour une percussion directe classique, sont rarement exploités de manière exhaustive, sans doute en raison des mauvais résultats obtenus. Ils portent en effet des traces de percussion très marquées mais certains éclats y sont encore attachés (pl. 1, 2, 3 - en annexe). D'autres, pourtant exploités sur le maximum de faces, présentent de nombreux accidents, de type Siret notamment (pl. 4 - en annexe). Les éclats obtenus sont souvent épais et fracturés transversalement, à talon aigu, pourvus de traces de percussion prononcées en partie proximale et distale (pl. 5 et 6 - en annexe). Ils n'ont pas servi de supports d'outils à l'abri Suard, très peu à l'abri Bourgeois-

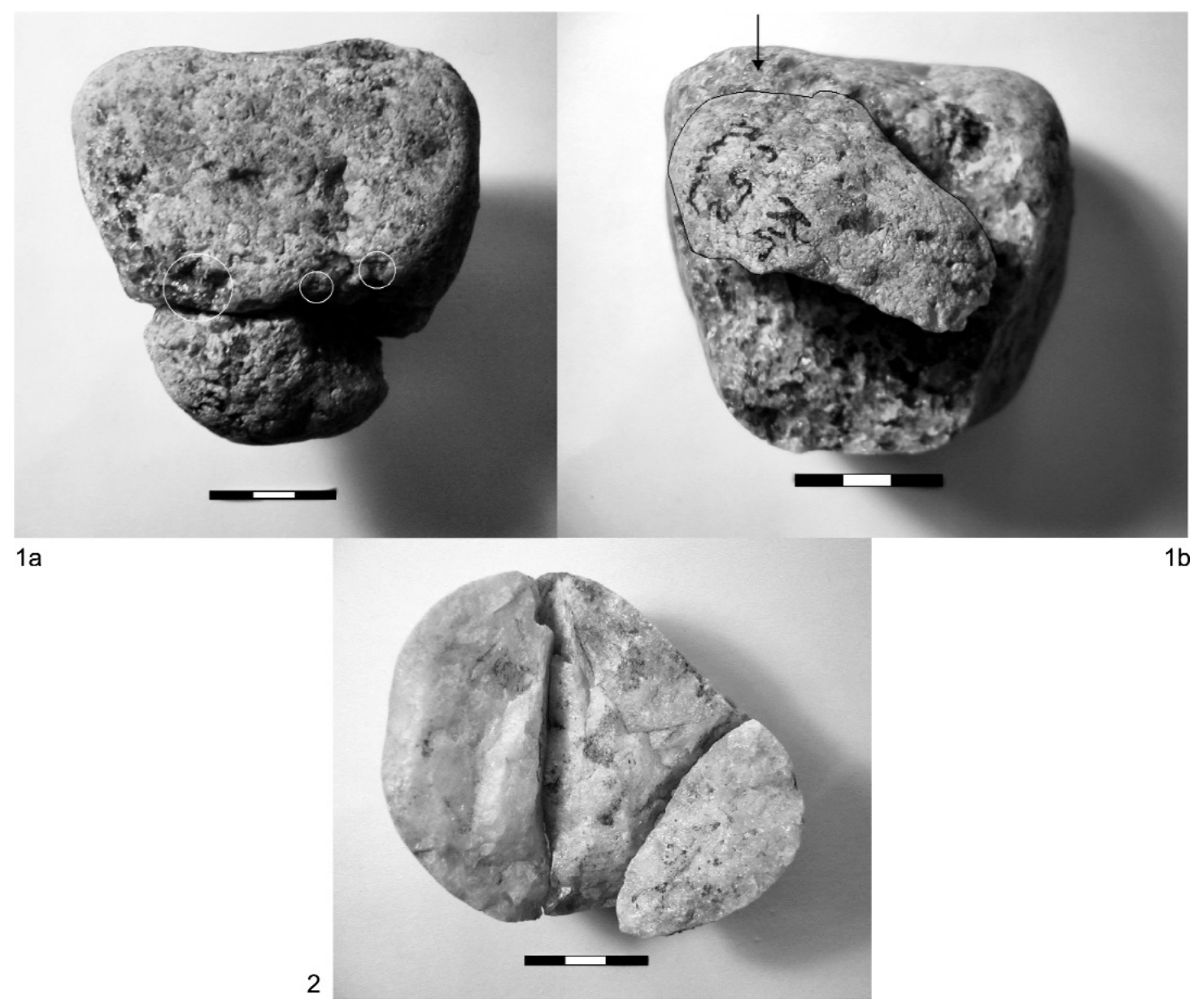

Figure 6 - Remontages découverts à l'abri Suard. 1. galet percuté sur enclume vue de dessus (1a) et vue de face (1b) ; 2. éclat bipolaire sur enclume fracturé par deux accidents de type Siret (quartzite).

Figure 6 - Refitting from Abri Suard. 1. pebble obtained by percussion on anvil, top (1a) and face (1b); 2. bipolar flake on anvil broken by two Siret accident (quartzite). 


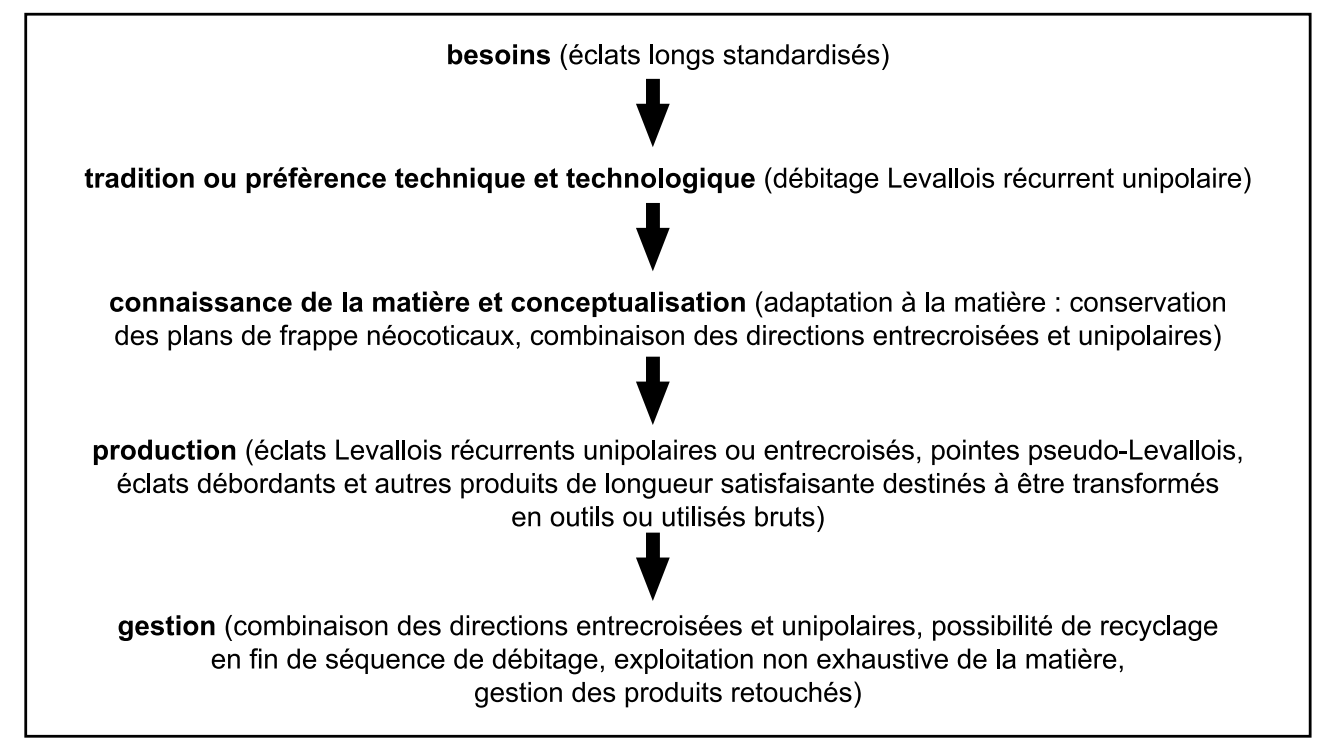

Figure 7 -

Comportement technoéconomique des Hommes de l'abri Suard.

Figure 7 - Technoeconomical behaviour, Suard rockshelter.

Delaunay, alors que les éclats indifférenciés issus d'un débitage classique l'ont été en très grande proportion. II semble donc que ces produits aient été débités pour être utilisés bruts et satisfaire des besoins immédiats.

II n'a été noté aucune évolution de la méthode entre l'abri Suard et l'abri Bourgeois-Delaunay qui, pourtant, présentent des changements techno-économiques évidents (Matilla
2004). A l'abri Suard, que ce soit sur le matériel en quartz ou en silex, l'essentiel de la production est issu du débitage Levallois récurrent unipolaire, avec une gestion de la matière première, depuis la récolte du matériau jusqu'à l'exploitation de l'outillage (fig. 7). La méthode de débitage bipolaire sur enclume complète, occasionnellement, la panoplie. A l'abri Bourgeois-Delaunay, les Hommes ont un comportement différent de ceux de Suard. La gestion de la matière

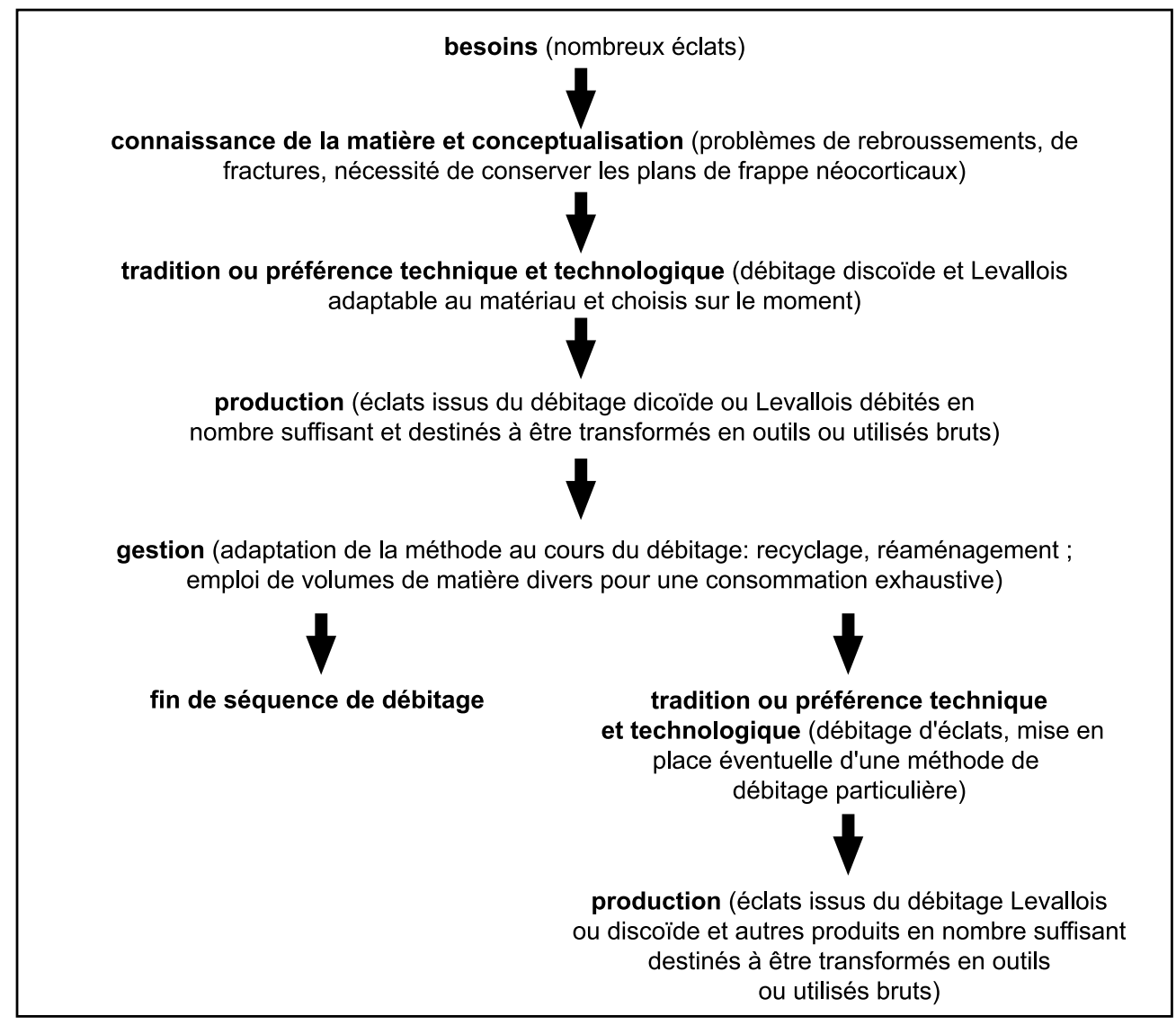

Figure 8 -

Comportement technoéconomique des Hommes de l'abri Bourgeois-Delaunay.

Figure 8 - Technoeconomical behaviour, $B-D$ rockshelter. 
première est beaucoup plus adaptée aux circonstances du débitage, et souvent exhaustive. Le débitage discoïde est majoritairement utilisé (fig. 8). La méthode de débitage bipolaire sur enclume est toujours employée et ne présente aucun changement. La présence de ce tronc commun entre les deux abris, alors que le comportement technique des Hommes évolue, est particulièrement intéressante. La percussion bipolaire sur enclume paraît être une méthode totalement indépendante de la production lithique principale. Elle n'intègre pas la logique de la chaîne opératoire menée dans chaque abri, qualitative à Suard, quantitative à Bourgeois-Delaunay, depuis la nature du besoin jusqu'à la production, en passant par l'expression d'une tradition ou d'une préférence technique et technologique (fig. 7 et 8) (Matilla 2004). II semble donc s'agir d'une méthode de débitage employée occasionnellement, pour un besoin immédiat en éclats de morphologie particulière, comme cela a été décrit à Mauran (Mourre 2004), indépendante de la chaîne opératoire principale. Elle ne guide en rien le comportement général des hommes vis-à-vis de leur production lithique.

\section{CONCLUSION}

La présence de plusieurs méthodes de débitage relevant de conceptions techniques et technologiques différentes et indépendantes révèle la richesse des connaissances des tailleurs et leur capacité d'adaptation en fonction de leurs besoins. La diversité des modalités d'application de la percussion directe au percuteur dur relève en effet de contraintes techniques, de choix techno-économiques et techno-typologiques particuliers. Dans le cas présent, la percussion bipolaire sur enclume ne semble être liée à aucune influence culturelle dans la mesure où, malgré le changement comportemental observé entre les deux abris vis-à-vis du matériel lithique, le débitage Levallois étant dominant à Suard, le discoïde à Bourgeois-Delaunay (Matilla 2004), la percussion bipolaire sur enclume est toujours utilisée. Elle semble donc être une méthode employée occasionnellement, pour un besoin immédiat en éclats.

Cette étude n'a pas de corollaire en Charente. II serait donc nécessaire d'obtenir des éléments de comparaison dans cette région afin de vérifier si l'emploi opportuniste de cette technique et méthode de débitage se répète, ou au contraire, s'il existe des cas où son utilisation peut être systématique.

\section{BIBLIOGRAPHIE}

BORDES F. 1947 - Étude comparative des différentes techniques de taille du silex et des roches dures. L'Anthropologie, 51, 1-2, 1947, p. 1-29.

BORDES F. 1961 - Typologie du Paléolithique ancien et moyen. Bordeaux : Delmas, 1961.

DEBENATH A. 1974 - Recherches sur les terrains quaternaires charentais et les industries qui leur sont associées ; Bordeaux : Université de Bordeaux 1, 1974, 2 vol. Thèse de doctorat d'Etat ès Sciences, $680 \mathrm{p}$.
DEBENATH A. et TOURNEPICHE J.F. éd. 1992 Neandertal en Poitou-Charentes. Association Régionale des Conservateurs des Musées du Poitou-Charentes, Poitiers, l'Isle d'Espagnac, Imprimeries Ebrard, 187 p.

DELAGNES A. 1992 - L'organisation de la production lithique au Paléolithique moyen. Approche technologique à partir de l'étude des industries de La Chaise-de-Vouthon (Charente), Paris : Université de Paris X, Thèse de Doctorat.

DURAN J.P. 2002 - Industries moustériennes en Languedoc, Roussillon et Catalogne au Würm ancien : la Combe, le Ratier, la Rouquette, les Anecs, Moutou-LaJoliette, la Caune de l'Arago, l'Arbreda ; Perpignan : Université de Perpignan. Thèse de doctorat.

JAUBERT J. et MOURRE V. 1996 - Coudoulous, Le Rescoundudou, Mauran : diversité des matières premières et variabilité des schémas de production d'éclats. Quaternaria Nova, 6, p. 313-341.

KUIJT I, PRENTISS W.C et PROKOTYLO D.L. 1995 Bipolar reduction : an experimental study of debitage variability. Lithic technology, 20, p. 116-127.

MATILLA K. 2001 - Contribution à l'étude des industries du Paléolithique moyen de l'abri Bourgeois-Delaunay (La Chaise-de-Vouthon, Charente). Le matériel en quartz et autres roches de la couche 9 (fouilles A. Debénath, 19671983) ; Perpignan : Université de Perpignan. DEA, 85 p.

MATILLA K. 2002 - Premiers résultats sur l'étude du matériel en quartz, en roches métamorphiques et magmatiques de gisements charentais du Paléolithique moyen. Abri Bourgeois-Delaunay (la Chaise-de-Vouthon, Charente), couche 9 (fouilles A. Debénath 1963-1983). Bulletin de la société préhistorique française, 99, p. 375-377.

MATILLA K. 2004 - Contribution à l'étude des industries de La Chaise-de-Vouthon (Charente). Techno-typologie et techno-économie du matériel sur galet. Fouilles David (1936-1961), fouilles A. Debénath (1967-1983) ; Perpignan : Université de Perpignan. Thèse de doctorat, $340 \mathrm{p}$.

MOURRE V. 1996 - Les industries en quartz au Paléolithique. Terminologie, méthodologie et technologie. Paléo, 8, p. 205-223.

MOURRE V. 1997 - Industries en quartz : précisions terminologiques dans les domaines de la pétrographie et de la technologie. Préhistoire et Anthropologie Méditerranéennes, 6, p. 201-210.

MOURRE V. 2004 - Le débitage sur enclume au Paléolithique moyen dans le sud-ouest de la France. In : P. Van Peer, D. Bonjean, et P. Semal (Eds.), Session 5 : Paléolithique moyen. Actes du XIVe Congrès de l'I.U.S.P.P., Liège (2-8 septembre 2001) Oxford, Archeopress, p. 2938, (B.A.R. International Series 1239). 
LA CHAISE-DE-VOUTHON (Charente), FOUILLES P. DAVID (1936-1961) ET FOUILLES A. DEBÉNATH (1967-1983) :

UN EXEMPLE DE DÉBITAGE BIPOLAIRE SUR ENCLUME

Karine MATILLA

\section{ANNEXES}




\section{Annexes - planches 1 à 6}

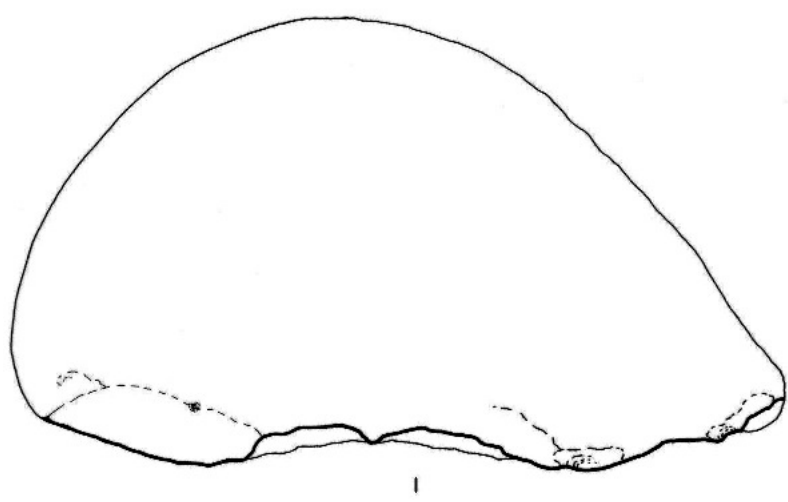

Planche 1 - Nucléus bipolaire sur enclume (quartzite).

Planche 1 - Bipolar core on anvil (quartzite).
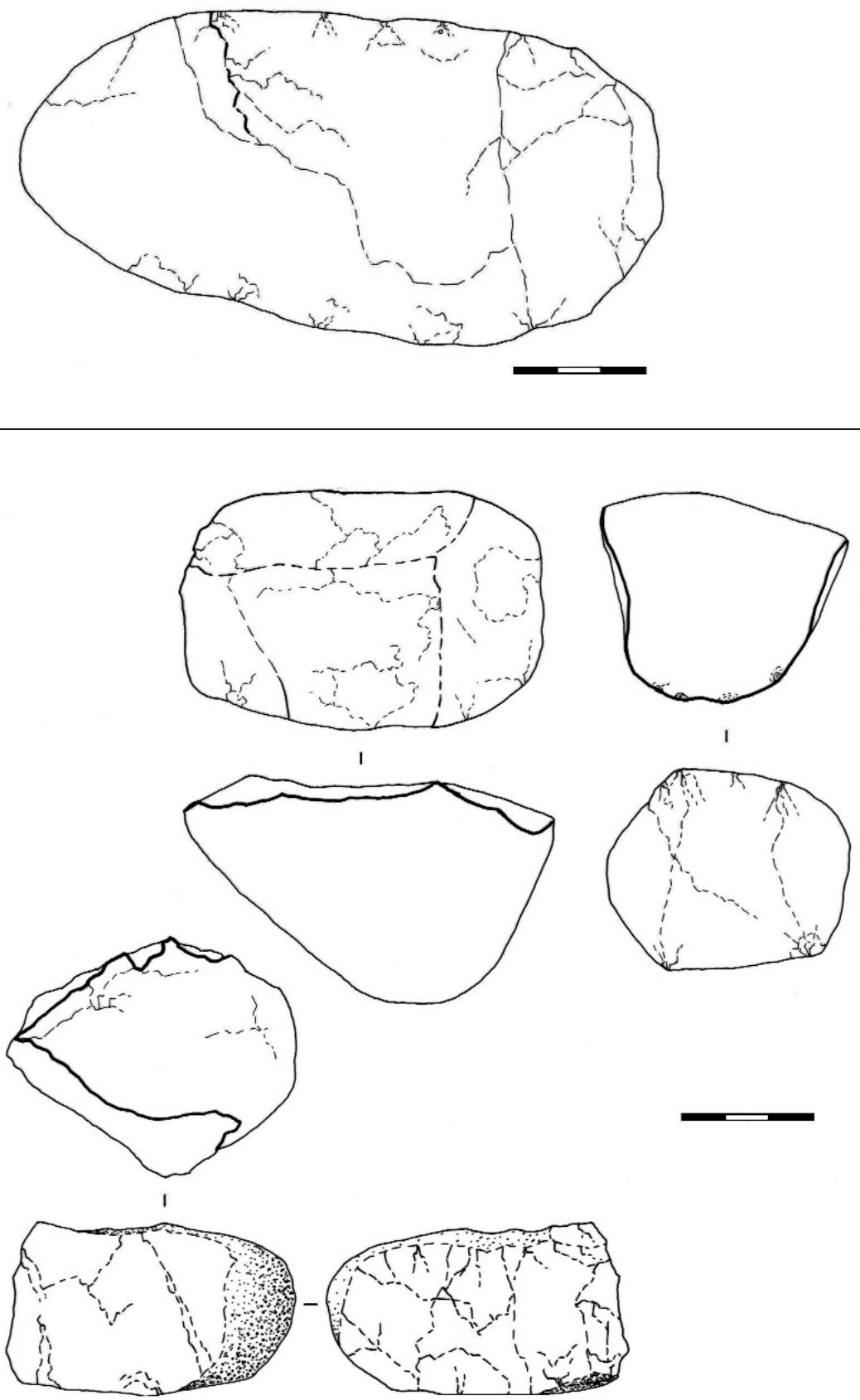

Planche 2 - Nucléus bipolaire sur enclume (quartzite).

Planche 2 - Bipolar core on anvil (quartzite). 

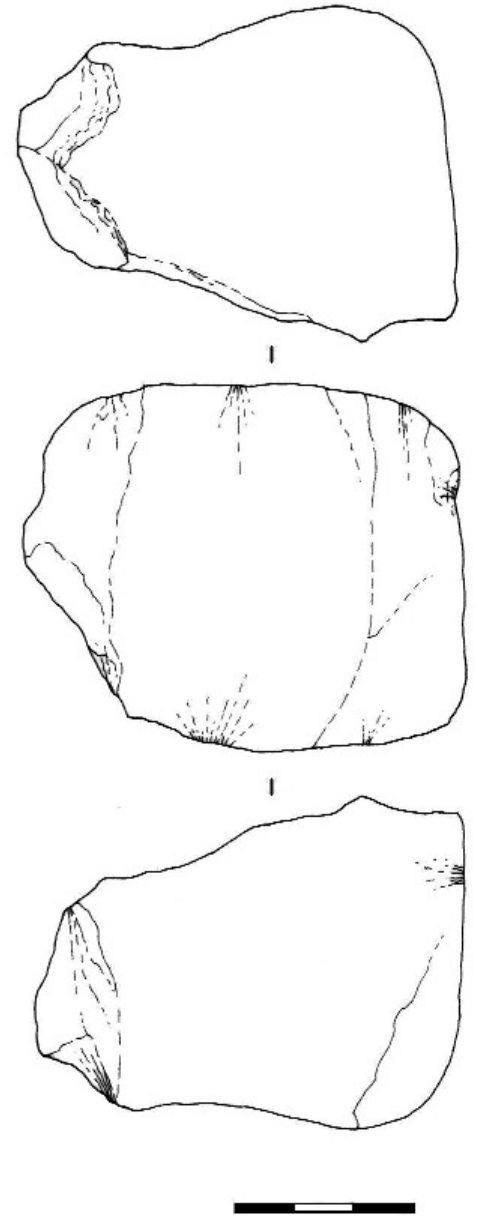

Planche 3 - Nucléus bipolaire sur enclume (quartzite).

Planche 3 - Bipolar core on anvil (quartzite).
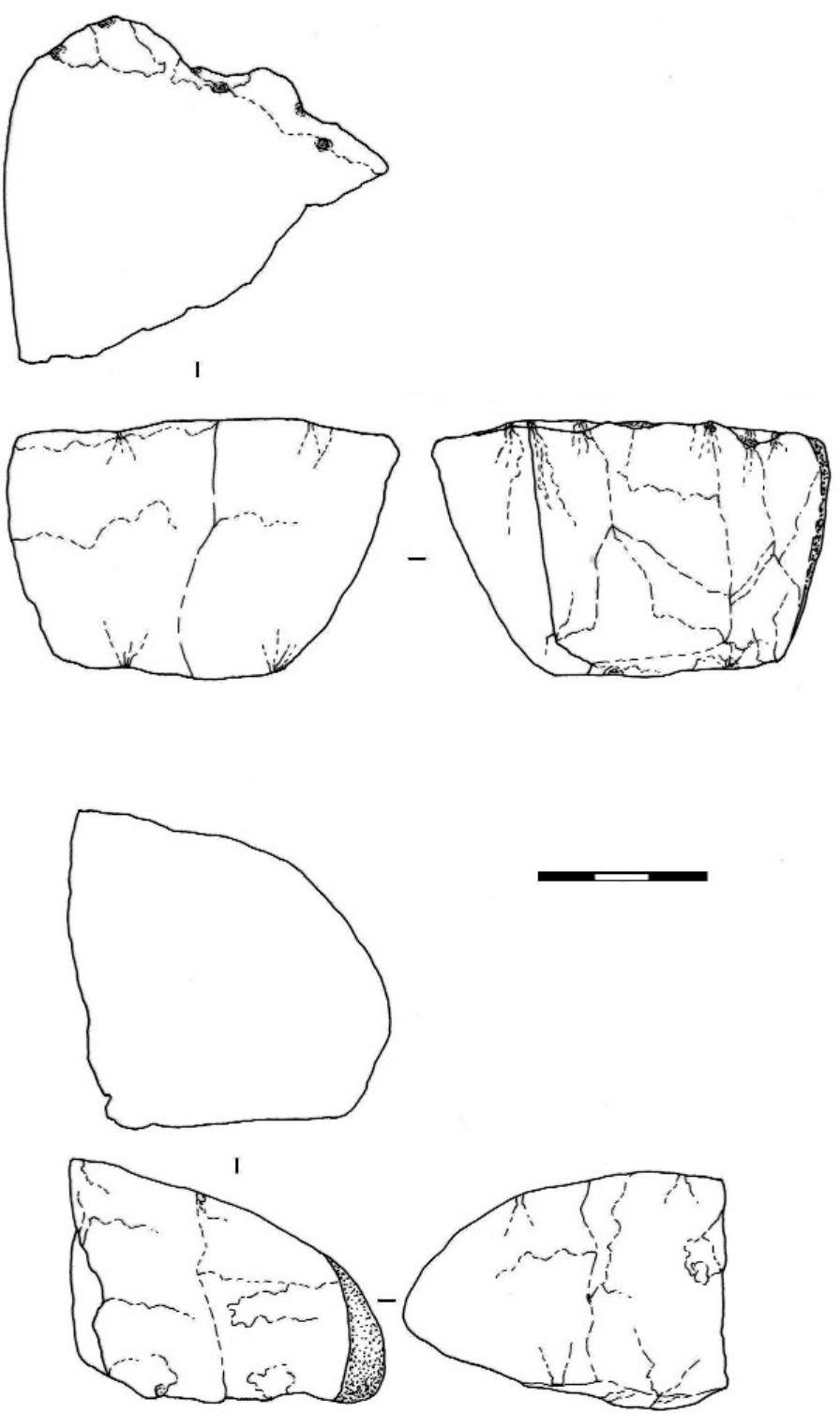

Planche 4 - Nucléus bipolaires sur enclume (quartzite).

Planche 4 - Bipolar cores on anvil (quartzite). 


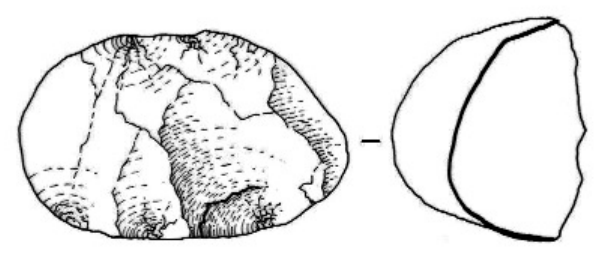

Planche 5 - Eclats bipolaires sur enclume de plein débitage (quartzite).

Planche 5 - Bipolar flakes of debitage on anvil (quartzite).
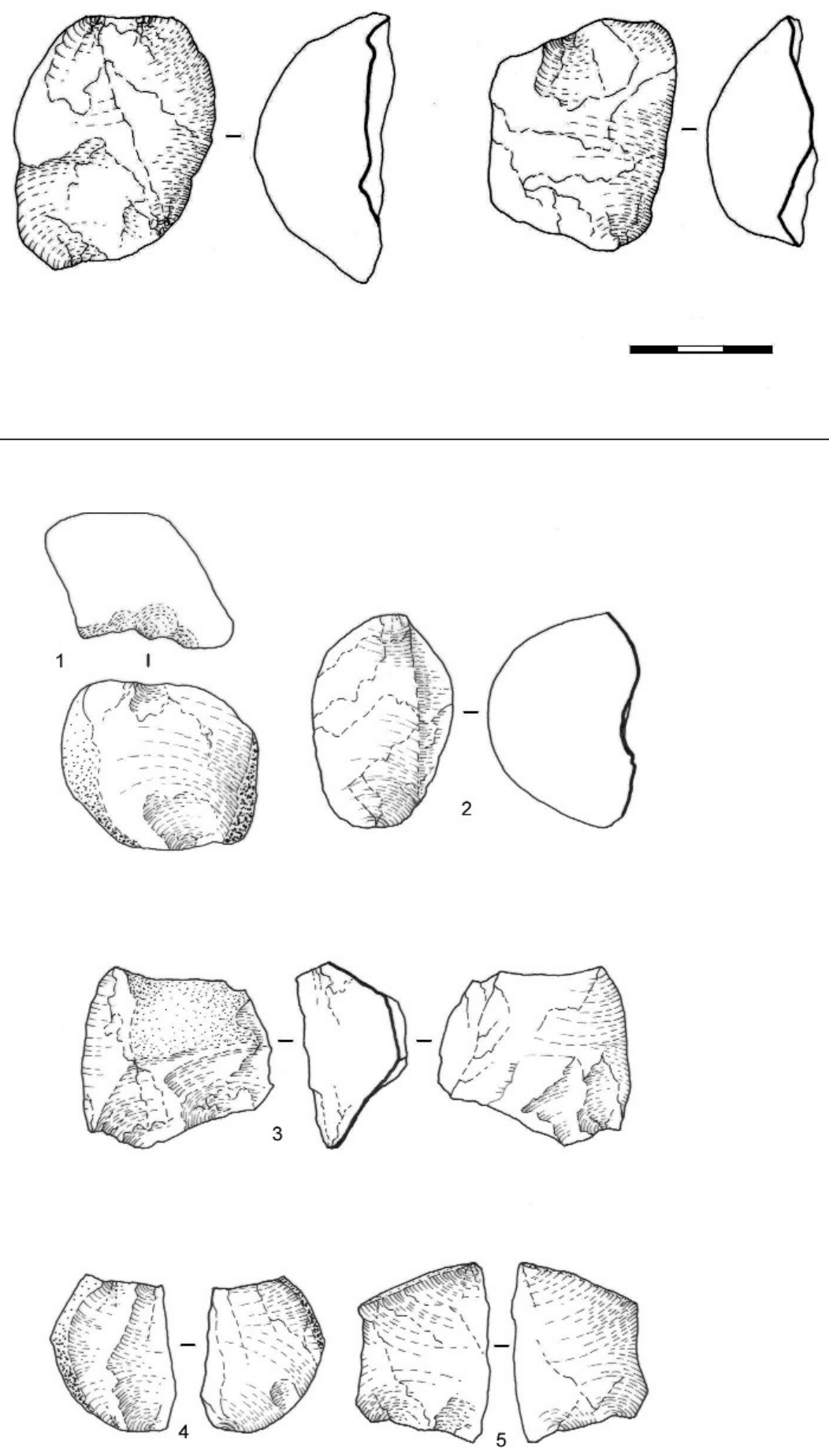

Planche 6 - 1 et 2, éclats bipolaires sur enclume d'entame ; 3,4, 5, éclats bipolaires sur enclume de plein débitage (quartzite).

Planche 6 - 1 and 2, first bipolar flakes on anvil ; 3, 4, 5, bipolar flakes of debitage on anvil (quartzite). 